\title{
Rice Bodies in a Patient with Oligoarticular Juvenile Idiopathic Arthritis
}

MICHIRO SUSA, MD, PhD; KEISUKE HORIUCHIㄹ, MD, PhD, Department of Orthopaedic Surgery, National Defense Medical College, Tokorozawa, Japan. Address correspondence to Dr. K. Horiuchi, Department of Orthopaedic Surgery, National Defense Medical College, 3-2 Namiki, Tokorozawa, Saitama 359-8513, Japan.E-mail: khoriuchi@ndmc.ac.jp. Written consent was provided from the patient's family for her case to be published anonymously. The ethics review board at the National Defense Medical College approved this report (\#3071). J Rheumatol 2019;46:1157-8; doi:10.3899/jrheum.181425

Oligoarticular juvenile idiopathic arthritis (JIA) is one of the most common subtypes of JIA, which, by definition, affects fewer than 5 joints during the course of the disease ${ }^{1}$. This condition typically involves the large joints of children and lacks systemic symptoms. Here we present a case of oligoarticular JIA that presented with characteristic intraarticular rice bodies.

A 5-year-old girl was brought to the orthopedic department with a swollen right knee, which her parents incidentally found a month prior. On presentation, she was apparently well without any systemic manifestations. Laboratory tests showed mild inflammation and elevation of antinuclear antibodies (positive at 1:320 dilution with homogeneous and speckled patterns). Tuberculosis skin test was negative. Magnetic resonance imaging examinations showed a large joint effusion with characteristic mosaic pattern (Figure 1). The joint was filled with small white fragments uniform in size and reminiscent of rice grains or fish eggs (Figure 2). Pathological analysis showed a prominent pannus formation and fibrinoid necrosis. Based on these clinical findings, a diagnosis of oligoarticular JIA was finally made. The fragmented hypertrophic synovium that underwent fibrinoid necrosis often results in the formation of rice bodies in the joint cavity. Rice bodies indicate the presence of chronic synovial inflammation, such as rheumatoid arthritis (RA) and tuberculosis arthritis. While rice bodies are relatively common in adult patients with RA, they are much less frequent in children ${ }^{2}$ and can often affect the tendons, leading to rupture ${ }^{3,4}$. The patient is currently treated with methotrexate and is well, without any sign of disease progression.

\section{REFERENCES}

1. Macaubas C, Nguyen K, Milojevic D, Park JL, Mellins ED. Oligoarticular and polyarticular JIA: epidemiology and pathogenesis. Nat Rev Rheumatol 2009;5:616-26.

2. Druschel C, Funk JF, Kallinich T, Lieb A, Placzek RP. Development of rice bodies in 2 children younger than 3 years. J Clin Rheumatol 2013;19:35-7.

3. Nabet A, Faruqui S, Hogan CJ. Rice bodies and a partial flexor tendon rupture in a patient with juvenile idiopathic arthritis: a case report and review of the literature. JBJS Case Connect 2017;7:e41.

4. Cuomo A, Pirpiris M, Otsuka NY. Case report: biceps tenosynovial rice bodies. J Pediatr Orthop B 2006;15:423-5.

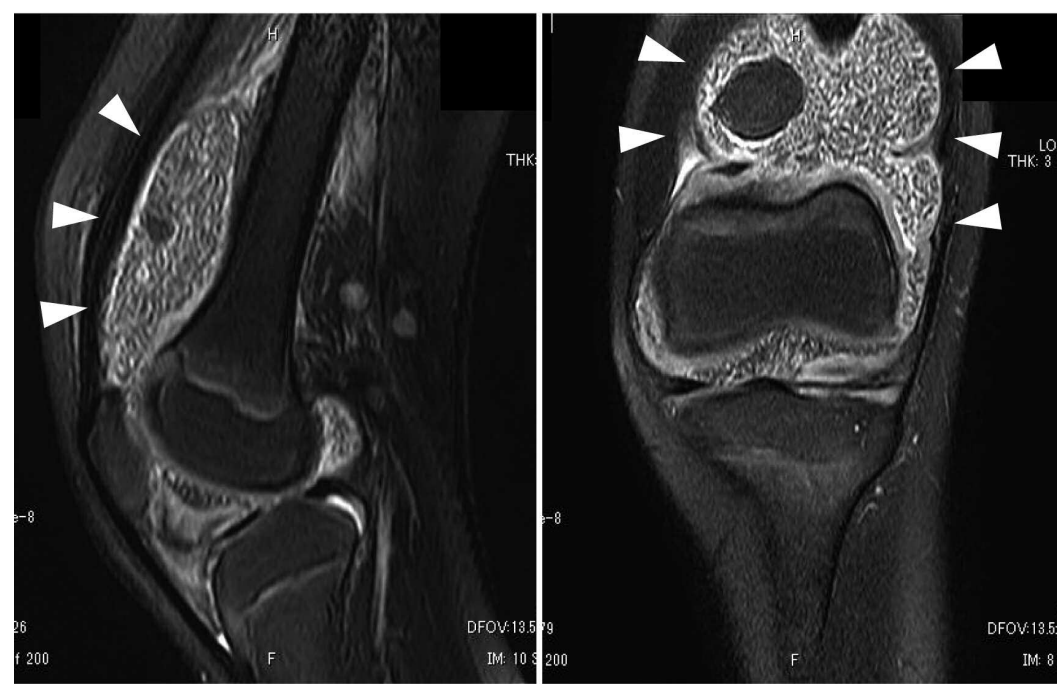

Figure 1. Fat-suppressed T2-weighted magnetic resonance images of the knee reveal tiny hypointense particles in the joint cavity (arrowheads). 

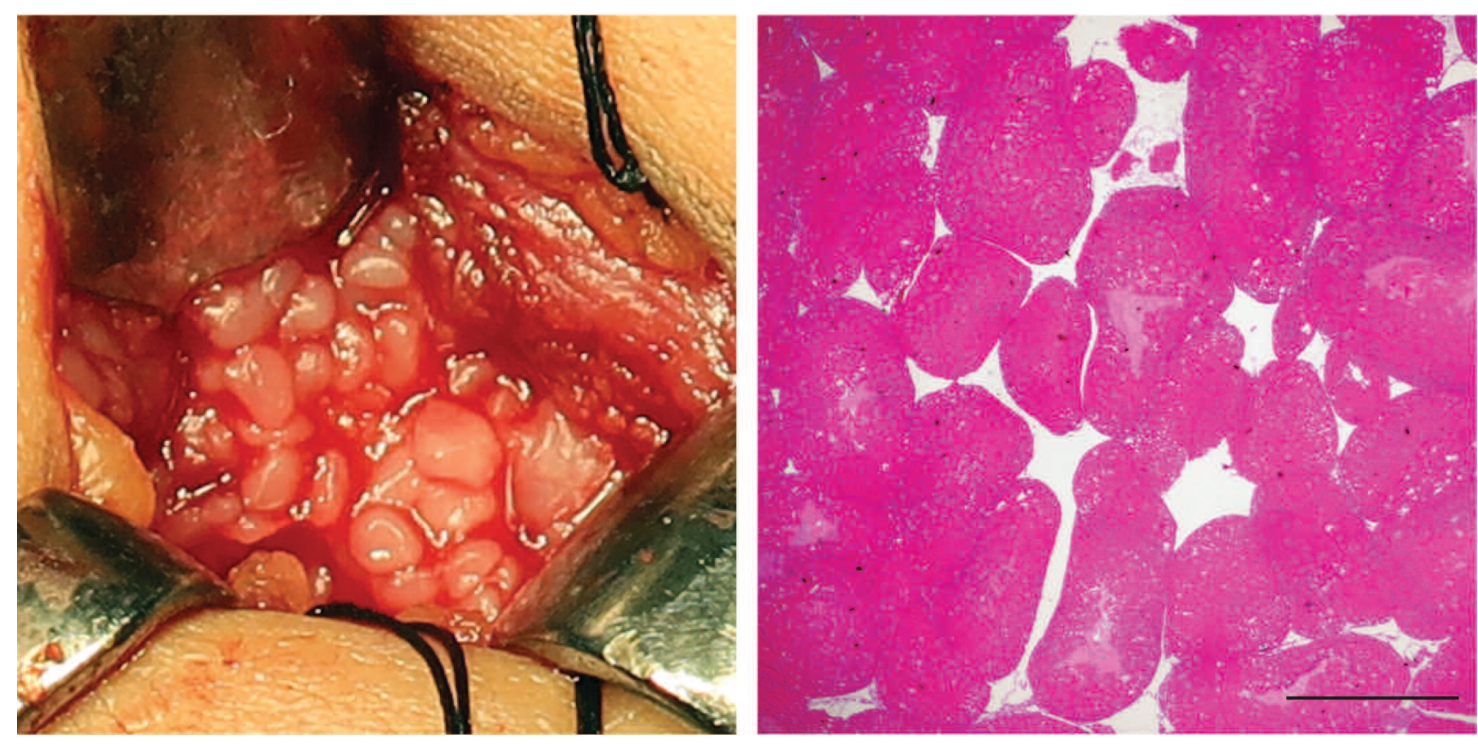

Figure 2. A photograph during minor surgery showing whitish grain-like bodies in the joint cavity (left panel). A hematoxylin-stained section of the rice bodies (right panel). Bar $=1 \mathrm{~mm}$. 